\title{
Generalized periodic discharges with triphasic morphology
}

\section{J. Andrew Hartshorn, MD' ; Brandon Foreman, MD, FACNS ${ }^{1,2}$}

'Department of Neurology and Rehabilitation Medicine, University of Cincinnati Medical Center, University of Cincinnati Gardner Neuroscience Institute, Cincinnati, $\mathrm{OH}$, USA

${ }^{2}$ Division of Neurocritical Care, University of Cincinnati Medical Center, Cincinnati, OH, USA

\begin{abstract}
Generalized periodic discharges (GPDs) with triphasic morphology are an electroencephalographic (EEG) pattern traditionally associated with encephalopathy and coma, although they have been observed in a wide array of neurological disorders. The clinical significance of these waveforms and their relationship to seizures and prognosis has been debated, and differentiation between interictal patterns, patterns associated with seizures, and patterns representing nonconvulsive status epilepticus can at times be a challenge. The most established literature suggests that GPDs, including those with triphasic morphology, are associated with the development of electrographic seizures, but that in the absence of clinical information, distinguishing waveforms based on morphology alone may not be clinically useful. Recent work has advocated for a more proactive approach in evaluating GPDs with triphasic morphology. Further studies of nonsedating antiseizure drugs in patients with GPDs with triphasic morphology that incorporate continuous EEG monitoring will be useful in tailoring therapy to optimize long-term clinical outcomes and recovery.
\end{abstract}

Keywords: Triphasic waves; Brain diseases; Generalized periodic discharges; Status epilepticus

\section{INTRODUCTION}

Generalized periodic discharges (GPDs) are electroencephalographic (EEG) waveforms that can be seen in a wide array of encephalopathies. By definition, they are repeated and generalized waveforms with relatively uniform morphology and duration, with a quantifiable interdischarge interval between consecutive waveforms, and recurrence of the waveform at nearly regular intervals (Fig. 1) [1]. There are different theories for their etiology and pathophysiology. Early work suggested that GPDs were due to widespread cortical destruction with relative sparing of white matter [2]. A more recent theory is that they result from either a synaptic failure of interneurons or impaired excitation of inhibitory interneurons, resulting in disinhibition of excitatory pyramidal cells. As a result of their frequent association with cardiac arrest and anoxic brain injury, it is thought that high-energy excitatory pyramidal cells may be more severely affected by hypoxic energy failure, resulting in disruption of feed forward inhibitory networks and propagation of GPDs [3]. The presence of GPDs is highly suggestive of a global encephalopathy and is seen in approximately $4 \%$ of patients in the hospital or intensive care unit setting undergoing EEG monitoring [4]. They are commonly associated

(C) 2019 The Korean Neurocritical Care Society

This is an Open Access article distributed under the terms of the Creative Commons Attribution Non-Commercial License (http://creativecommons.org/licenses/by-nc/4.0/) which permits unrestricted noncommercial use, distribution, and reproduction in any medium, provided the original work is properly cited. 


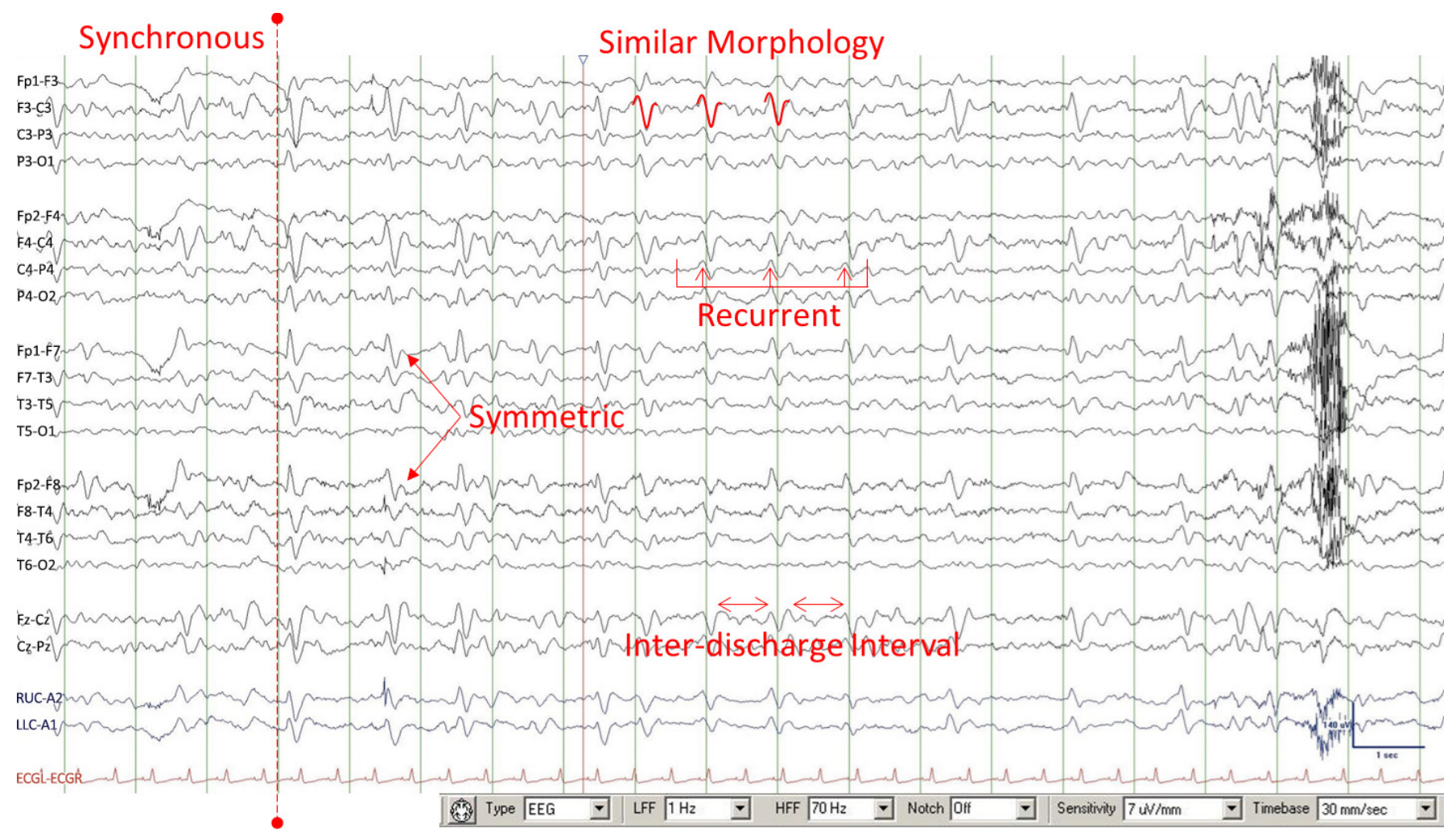

Fig. 1. Generalized periodic discharges: a 51-year-old man with a history of immunosuppression, liver disease, and heart failure with hyponatremia and sepsis. This pattern is described as a $1 \mathrm{~Hz}$ generalized periodic discharge pattern; note the features labeled in the figure that constitute this pattern.

with toxic/metabolic encephalopathy, anoxia, hypothermia, infections, acute neurological injury, and nonconvulsive status epilepticus (NCSE) [5].

Triphasic waves (TWs) are likewise generalized and periodic EEG waveforms seen in a wide array of encephalopathies. TWs have specific characteristics that are thought to distinguish them from other forms of GPDs. TWs are classically described as having an initial sharp negative deflection, followed by a prominent positive deflection, and then a negative deflection. They tend to be moderate to high amplitude ( 100 to $300 \mu \mathrm{V}$ ) with a frequency of 1.5 to $2.5 \mathrm{~Hz}$. Their location tends to be predominantly frontal (although less frequently occipital and temporal) with an anterior to posterior time lag (Table 1, Fig. 2). However, both GPDs and TWs overlap substantially with regard to their morphology and clinical associations, and the 2012 American Clinical Neurophysiology Society (ACNS) standardized EEG terminology has established the term "GPDs with triphasic morphology (GPD+TW)" [1] to replace the traditional term 'triphasic waves.'

GPD+TW were first described in 1955, when Adams and Foley [6] noted blunt spike-and-slow wave complexes among a subset of patients with liver disease [6]. These were subsequently giv-
Table 1. Morphologic features traditionally thought to distinguish waveforms $[5,22,28,29]$

\begin{tabular}{|c|c|c|}
\hline Feature & GPD+TW & $\begin{array}{l}\text { GPD associated } \\
\text { with seizure }\end{array}$ \\
\hline Duration of phase I of waveform & Longer & Shorter \\
\hline Duration of entire waveform & Longer & Shorter \\
\hline Angles between phases & Larger (blunted) & Smaller (sharp) \\
\hline Amplitude of phase II & Higher & Lower \\
\hline Location & Frontrocentral & Frontopolar \\
\hline Discharge frequency & $\leq 2.5 \mathrm{cycles} / \mathrm{sec}$ & $>1 \mathrm{~Hz}$ \\
\hline Extraspike components & No & Yes \\
\hline Background slowing & More & Less \\
\hline Likelihood of all three phases & Yes & No \\
\hline Dominant 1st phase & No & Yes \\
\hline Negative polarity ${ }^{\text {a) }}$ & No & Yes \\
\hline Anterior-posterior lag & Yes & No \\
\hline Increased with stimulation & Yes & No \\
\hline
\end{tabular}

GPD, generalized periodic discharge; TW, triphasic wave.

a) Polarity refers to the dominant phase of the discharge.

en the name 'triphasic waves' by Bickford and Butt [7] in 1955, who studied EEG patterns of patients with hepatic coma. 


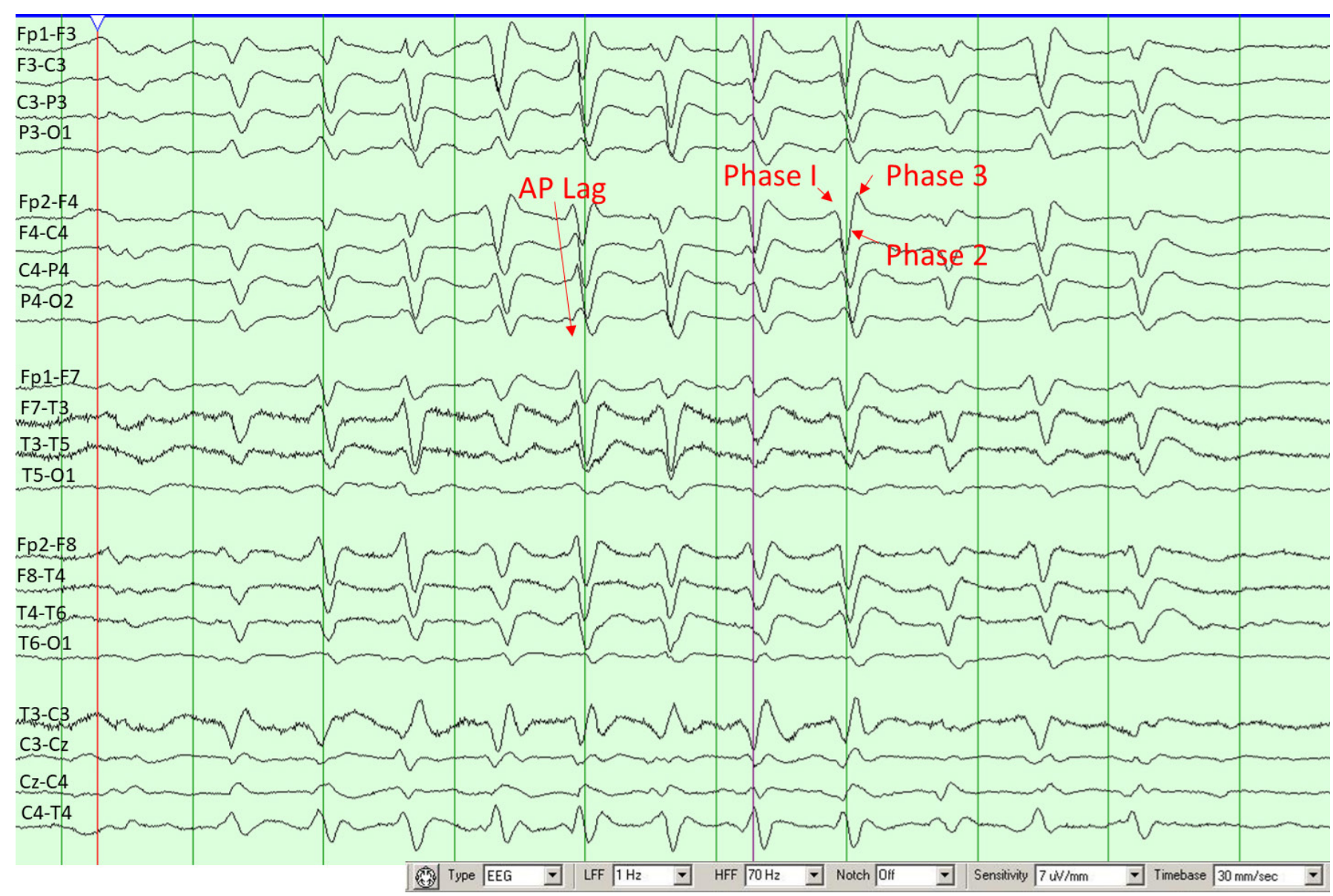

Fig. 2. Generalized periodic discharges with triphasic morphology: a 59-year-old man with liver diease who presented with encephalopathy and an elevated ammonia. Distinctive features include three phases and an anterior-posterior time lag as shown in the figure.

Throughout multiple stages of mental status decline, they noted clusters of GPD+TW followed by relative quiescence, although they seemed to be more frequent in deeper stages of coma. Consequently, it was suggested that GPD+TW were a measure of severity of hepatic coma and their presence could point towards a worse prognosis [7].

Although thought to be highly specific for hepatic dysfunction, further work showed that GPD+TW were present across a wide spectrum of disorders. They have been documented in patients with azotemia, hypoxia, hyperosmolarity, and hypoglycemia [8], and indeed, metabolic derangements appear to be a common etiology for GPD+TW $[9,10]$. Beyond these, GPD+TW have been observed in Alzheimer's disease [11] and multiple drug intoxications (baclofen [12], levodopa [13], lithium [14], ifosfamide [15], and metrizamide [16]). In addition, GPD+TW have very infrequently been seen in structural lesions, including brain stem-diencephalic lesions [17].
The exact origin of GPD+TW has been difficult to elucidate, but the predominant theory is that they result from dysfunction of the thalamocortical circuits [8]. Source localization techniques were used to understand the pathophysiology of GPD+TW in a study involving 12 patients. The density of GPD +TW was found mainly in the bilateral medial frontal regions along the cingulate cortices, making it reasonable to hypothesize that the medial frontal area plays a role in the generation of these waveforms [18]. In a study of patients with GPD+TW that examined radiographic correlates, GPD+TW were associated with the presence of white matter abnormalities [19]. However, the overlap between GP$\mathrm{D}+\mathrm{TW}$ and GPD without triphasic morphology may mean that both thalamocortical circuits and cortical dysfunction are required for their formation. The necessary and sufficient biological substrate for the development of GPDs and particularly GP$\mathrm{D}+\mathrm{TW}$ has not been fully elucidated. 


\section{UPDATED TERMINOLOGY AND INTERRATER AGREEMENT}

In 2012, the ACNS released guidelines in an attempt to provide uniformity in reporting EEG, with a particular focus on abnormal periodic and rhythmic patterns encountered most commonly in critically ill patients. GPDs can vary based on location (e.g., frontally predominant, occipitally predominant, midline predominant, generalized, or not otherwise specified) and frequency among a variety of additional modifiers (Table 2) [1].

These guidelines have been helpful in providing more uniformity in EEG reporting, although there continues to be some variability in the interpretation and reporting of GPD+TW. In 2014, interrater agreement (IRA) of the ACNS' standardized terminology was examined and showed that for most terms, IRA was high. Forty-nine raters showed almost perfect agreement for seizures, terms 1 and 2 (e.g., generalized and periodic), and modifiers describe sharpness, amplitude, frequency, and the number of phases. However, the IRA for 'triphasic morphology' was only moderate (58\%) [20]. This was similar to a study examining the IRA for EEGs of comatose patients in general [21], as well as a study specifically examining GPDs that found that among 20 patients, the IRA for 'triphasic morphology' was only fair ( $\kappa$ of 0.33 ) [22]. In a retrospective cohort study of 92 patients with GPDs, the IRA for 'triphasic morphology' was "substantial," with a $\kappa$ of 0.67 [23]. However, this study utilized only two raters, where the prior IRA study for GPDs used 11 to rate EEGs; this difference could have contributed to the disparity in the IRA.

\section{NONCONVULSIVE STATUS EPILEPTICUS AND SEIZURES}

When GPDs are found, with or without triphasic morphology, it is always important to consider first whether or not they represent an ictal rhythm [5]. At any frequency and with any morphology, the appearance of motor manifestations, including myoclonic or rhythmic muscle jerking in conjunction with GPDs, indicates that the pattern is ictal, and both clinical and electrographic manifestations should be treated as with any seizure. Broadly, the diagnosis of nonconvulsive seizures or NCSE is given in patients without known epileptic encephalopathy whose EEG shows epileptiform discharges greater than $2.5 \mathrm{~Hz}$, or if less than or equal to $2.5 \mathrm{~Hz}$, also demonstrate EEG and clinical improvement after intravenous (IV) antiseizure drugs (ASDs) or subtle clinical ictal phenomena or typical spatiotemporal evolution. For patients with known epileptic encephalopathy, there should be an increase in prominence or frequency of epileptiform discharges from baseline as well as electrographic and clinical improvement with IV ASDs [24]. This distinguishes patterns that are slower than $2.5 \mathrm{~Hz}$ without motor manifestations and without evolution or response to ASD, such as cefepime-induced encephalopathy.

There have been attempts to better classify whether or not GP$\mathrm{D}+\mathrm{TW}$ in a comatose patient represent an ictal rhythm when uncertainty exists. In a retrospective study of two groups of patients

Table 2. American Clinical Neurophysiology Society GPDs modifiers

1. Prevalence: how much of the record per epoch contains the GPDs

2. Duration: how long dose the GPD activity continues during the recording, in minutes or hours

3. Frequency: the number of GPDs occurring in 1 second

4. Number of phases: the number of baseline crossings in a typical GPD waveform

5. Sharpness: the time in milliseconds for the sharpest and the most prominent phase of the GPD

6. Amplitude: the highst amplitude of the GPD waveform in an anterior-posterior bipolar montage should be measured; amplitude of the GPD can also be measured relative to the background activity.

7. Polarity: whether the highest amplitude of the GPD is negative, positive, or unclear

8. Stimulus induced: whether the GPDs occur spontaneously or are induced with a stimulus

9. Evolving or fluctuating: whether the GPDs change in frequency, morphology, or location (evolution) or whether the changes are present but not enough to be classified as evolving (fluctuating)

10. Plus: whether additional features make the GPD pattern appear more epileptiform

Other minor modifiers can also be included when describing GPDs. These include the following terms:

1. Quasi: used to modify the rhythmic or periodic nature of the GPDs and only if determined by quantitative computer analysis (not by visual impression)

2. Sudden or gradual onset: used to describe how GPDs appear, suddenly (previously called paroxysmal) or over several seconds

3. Triphasic morphology: used to describe the shape of the GPDs

4. Lag of waveforms: either an anterior to posterior or a posterior to anterior lag may be seen in various components of the GPD

GPD, generalized periodic discharge. 
with decreased consciousness, differences between GPD+TW and generalized NCSE (GNSCE) were evaluated morphologically. Among 87 EEGs with GPD+TW and 27 EEGs with GNCSE, they found several different characteristics, the most significant being that epileptiform discharges associated with GNCSE had higher frequency, shorter duration of phase one and less generalized background slowing. Administration of benzodiazepines caused a marked decrease of epileptiform discharges, with cessation of GNCSE in seven of the eight patients. These same medications were administered to two patients in the GPD+TW group and there were no significant or electrographical or clinical changes. Interestingly, they also found that auditory and/or noxious stimuli caused immediate increase in discharges among patients with GP$\mathrm{D}+\mathrm{TW}$, while having no effect on epileptiform discharges in the GNSCE group. In a small set of patients, well-defined sleep was detected and GPD+TW were absent during this period [25].

In the link between GPD+TW and NCSE, other authors have advocated for differentiation between "typical" and "atypical" TW. Atypical TW have been defined as "localized or lateralized sharp waves with triphasic configuration," and when found in patients with altered mental status or depressed consciousness are more suggestive of NCSE [26]. In this case, these atypical 'triphasic waves' might better be classified as lateralized periodic discharges (LPDs), according to ACNS guidelines, rather than GP$\mathrm{D}+\mathrm{TW}$. Unfortunately, criteria to firmly differentiate ictal from interictal GPD patterns have not been validated [27].

GPDs with or without TW that do not fulfill the criteria for nonconvulsive seizures or NCSE remain highly associated with the development of electrographic seizures. In a matched case-control of patients with and without GPDs, nonconvulsive seizures were seen in $26.5 \%$ (vs. $8 \%$ in controls without GPDs), of whom nearly half had focal seizures. Seizures occurred after the development of GPDs in $42 \%$ and in $14.5 \%$ of patients, seizures occurred after 48 hours [4]. In a cohort of 4,772 patients undergoing continuous EEG (cEEG) interpreted by experienced raters using the ACNS standardized terminology, LPDs, lateralized rhythmic delta activity, and GPDs were associated with seizures, while generalized rhythmic delta activity was not. Among GPDs specifically, those with frequencies between 1.5 to $2 \mathrm{~Hz}$ had a higher association with development of seizures (odds ratio, 2.31; 95\% confidence interval, 1.25 to 4.11 ), suggesting that longer EEG monitoring may be warranted when GPDs are encountered [28].

Morphologic features do not appear to accurately stratify GPDs based on their association seizures. One retrospective study found that the amplitude and duration of the waveform were associated with the development of seizures or status epilepticus, but this was not specific enough for clinical purposes [29]. In an interrater agreement study, raters judged GPDs with or without TW based on their clinical knowledge. Raters tended to judge a waveform as 'triphasic' based on traditional morphologic features (Table 1) and in the absence of clinical information, agreed $93 \%$ of the time that seizures would develop based purely on their assessment of the waveform. However, this correlated only weakly with the actual appearance of seizures; in fact, both GPDs and GPD+TW were equally likely to be associated with seizures regardless of waveform or the presence of classically 'triphasic' features such as anterior-posterior time lag [22]. Fig. 2 demonstrates a classic 'triphasic wave' pattern $(\mathrm{GPD}+\mathrm{TW})$ in a patient with hepatic failure; within 24 hours of this recording, he developed focal seizures arising from the right parietal region that were subsequently treated with two conventional ASDs.

In another retrospective cohort study, 92 patients were divided in two groups: those with seizures and those without seizures. The cEEG data and clinical features were then analyzed to predict the development of seizures. Variables with a statistically significant risk of having seizures included focality on EEG, interburst suppression, history of epilepsy, an abnormal neuroimaging test, and generalized pattern without triphasic morphology. GPD+TW had a statistically significant decreased risk of seizures [23]. However, others have noted that their definition of GP$\mathrm{D}+\mathrm{TW}$ does not appear in line with ACNS guidelines and this may have influenced their findings [30].

\section{CLINICAL OUTCOMES}

The presence of GPDs with or without TW was historically thought to be a poor prognostic marker. A retrospective case-control study was performed involving 200 patients with GPDs and 200 controls matched by age, etiology, and level of consciousness. GPDs were not independently associated with worse outcome, whereas coma, sepsis, cardiac arrest, and NSCE were associated with worse outcome after matching for controls [4].

In the postcardiac arrest setting, GPDs are commonly encountered; however, prognosis in these cases may be linked to the presence or absence of background EEG activity. GPDs on a completely suppressed background have been associated with worse outcomes, whereas if they surface out of a normal background EEG, the prognosis was improved [31]. In 47 patients with postanoxic encephalopathy and generalized epileptiform activity, those with good clinical outcomes (defined by a score of 1 to 2 on the cerebral performance category) had higher background continuity, lower relative discharge power, and lower discharge periodicity [32]. This suggests that the relationship between the GPDs and their background is critically important in prognostic interpretation. 
Interestingly, the same may be true in nonhypoxic coma. In a 9-year cohort study evaluating outcomes among patients with acute encephalopathy and TW excluding cardiac arrest, a lack of EEG background reactivity was independently associated with death [19]. Interestingly, in the case-control study of patients with and without GPDs, there was a statistically significant association between GPDs and mortality when patients with cardiac arrest were excluded [4]. Background reactivity has been shown to be prognostically important in both nonanoxic [33] and anoxic [34] patient populations. There is debate about whether or not GPDs after cardiac arrest represent ischemic brain injury (i.e., are an epiphenomenon, or create further injury through an imbalance between the supply and demand of the injured brain). The Treatment of Electroencephalographic Status Epilepticus After Cardiopulmonary Resuscitation (TELSTAR) trial, which is currently in the recruitment phase, will assess postarrest patients with status epilepticus including GPDs and their clinical neurological outcomes, randomizing them to either receive aggressive medical treatment with ASDs to suppress all epileptiform activity or best medical care [35].

\section{TREATMENT AND FUTURE DIRECTIONS}

Recent work has found that the use of short-acting benzodiazepines or nonsedating ASDs may be useful to discern the potentially ictal nature of periodic or rhythmic patterns that do not fulfill criteria for nonconvulsive seizures or NCSE, such as GPD+TW. In a retrospective case series of patients with GPD+TW, $18.9 \%$ of patients had positive clinical responses to a benzodiazepine, compared to $42.2 \%$ who responded after a trial of nonsedating ASDs such as levetiracetam. Positive responses were defined as a resolution of EEG pattern and either unequivocal improvement in encephalopathy or appearance of previously absent normal EEG patterns. In addition, responses were categorized by time frameimmediate, delayed ( $>2$ hours) but unequivocal, delayed equivocal (cases where improvement could potentially bet attributed to something other than the ASD trial) or no response. Patients who responded to benzodiazepines did so immediately, whereas patients receiving nonsedating ASDs tended to have delayed responses [36]. Fig. 1 above shows a GPD pattern that was treated with $1 \mathrm{mg}$ lorazepam after which there was clear clinical improvement, constituting a positive response and suggesting that the pattern represented an ictal discharge warranting treatment.

A rapidly-acting nonsedating ASD could be of particular benefit in patients who either can't receive benzodiazepines or who don't initially respond to benzodiazepines. Consideration should be given to the time course of responses, as there is often overlap between NCSE and toxic-metabolic encephalopathies and improvement cannot always be attributed definitively to the nonsedating ASD trial. "ASD responsiveness" may be a useful descriptor among these GPD+TW patients. In contrast, others have argued that the risk of a benzodiazepine and/or nonsedating ASD for all patients with GPD+TW opens up risks for unnecessary medication overuse, side effects, interactions and potentially higher mortality and longer hospital stays [37]. The decision to treat should follow an individualized approach; recognition of the association between GPD+TW and seizures should raise concern and warrant at the very least serial or CEEG monitoring.

\section{CONCLUSION}

GPD+TW continue to be a debated pattern. Their association with seizures is now clear, but the interpretation of GPDs as having 'triphasic morphology' and the perception that these are not associated with seizures is often subjective, based on clinical history rather than the objective nature of the discharges. The presence of GPD+TW should prompt additional monitoring, ideally using cEEG and may, when uncertainty exists, warrant evaluation using a short-acting benzodiazepine or nonsedating ASD in order to discern the effects of the pattern on the patient's clinical exam and EEG. Ultimately, GPD + TW likely do not contribute to poor outcome independent from the patient's underlying diagnosis; however, seizures likely do contribute and; therefore, the two entities should be distinguished by clinicians wherever possible.

\section{ARTICLE INFORMATION}

\section{Conflict of interest}

No potential conflict of interest relevant to this article.

\section{ORCID}

J. Andrew Hartshorn, https://orcid.org/0000-0002-5495-0170

Brandon Foreman, https://orcid.org/0000-0002-5418-674X

\section{Author contributions}

Conceptualization: BF. Data curation \& Formal analysis: JAH and BF. Visualization \& Writing-original draft: JAH. Writing- review editing: JAH and $\mathrm{BF}$.

\section{REFERENCES}

1. Hirsch LJ, LaRoche SM, Gaspard N, Gerard E, Svoronos A, Herman ST, et al. American Clinical Neurophysiology Society's standardized critical care EEG terminology: 2012 version. J 
Clin Neurophysiol 2013;30:1-27.

2. Gloor P, Kalabay O, Giard N. The electroencephalogram in diffuse encephalopathies: electroencephalographic correlates of grey and white matter lesions. Brain 1968;91:779-802.

3. van Putten MJ, Hofmeijer J. Generalized periodic discharges: pathophysiology and clinical considerations. Epilepsy Behav 2015;49:228-33.

4. Foreman B, Claassen J, Abou Khaled K, Jirsch J, Alschuler DM, Wittman J, et al. Generalized periodic discharges in the critically ill: a case-control study of 200 patients. Neurology 2012;79:195160.

5. Sully KE, Husain AM. Generalized periodic discharges: a topical review. J Clin Neurophysiol 2018;35:199-207.

6. Adams RD, Foley JM. The neurological disorder associated with liver disease. Res Publ Assoc Res Nerv Ment Dis 1953;32:198237.

7. Bickford RG, Butt HR. Hepatic coma: the electroencephalographic pattern. J Clin Invest 1955;34:790-9.

8. Karnaze DS, Bickford RG. Triphasic waves: a reassessment of their significance. Electroencephalogr Clin Neurophysiol 1984;57:193-8.

9. Bahamon-Dussan JE, Celesia GG, Grigg-Damberger MM. Prognostic significance of EEG triphasic waves in patients with altered state of consciousness. J Clin Neurophysiol 1989;6:3139.

10. Brenner RP. The interpretation of the EEG in stupor and coma. Neurologist 2005;11:271-84.

11. Sundaram MB, Blume WT. Triphasic waves: clinical correlates and morphology. Can J Neurol Sci 1987;14:136-40.

12. Hormes JT, Benarroch EE, Rodriguez M, Klass DW. Periodic sharp waves in baclofen-induced encephalopathy. Arch Neurol 1988;45:814-5.

13. Neufeld MY. Periodic triphasic waves in levodopa-induced encephalopathy. Neurology 1992;42:444-6.

14. Kemperman CJ, Notermans SL. Creutzfeld-Jacob like syndrome due to lithium toxicity.J Neurol Neurosurg Psychiatry 1989;52:291.

15. Wengs WJ, Talwar D, Bernard J. Ifosfamide-induced nonconvulsive status epilepticus. Arch Neurol 1993;50:1104-5.

16. Drake ME, Erwin CW. Triphasic EEG discharges in metrizamide encephalopathy.J Neurol Neurosurg Psychiatry 1984;47:324-5.

17. Aguglia U, Gambardella A, Oliveri RL, Lavano A, Quattrone A. Nonmetabolic causes of triphasic waves: a reappraisal. Clin Electroencephalogr 1990;21:120-5.

18. Kwon OY, Jung KY, Park KJ, Kang JK, Shon YM, Lee IK, et al. Source localization of triphasic waves: implications for the pathophysiological mechanism. Clin EEG Neurosci 2007;38:161-7.

19. Sutter R, Stevens RD, Kaplan PW. Significance of triphasic waves in patients with acute encephalopathy: a nine-year cohort study. Clin Neurophysiol 2013;124:1952-8.

20. Gaspard N, Hirsch LJ, LaRoche SM, Hahn CD, Westover MB; Critical Care EEG Monitoring Research Consortium. Interrater agreement for critical care EEG terminology. Epilepsia 2014;55:1366-73.

21. Young GB, McLachlan RS, Kreeft JH, Demelo JD. An electroencephalographic classification for coma. Can J Neurol Sci 1997;24:320-5.

22. Foreman B, Mahulikar A, Tadi P, Claassen J, Szaflarski J, Halford JJ, et al. Generalized periodic discharges and 'triphasic waves': a blinded evaluation of inter-rater agreement and clinical significance. Clin Neurophysiol 2016;127:1073-80.

23. Alkhachroum AM, Al-Abri H, Sachdeva A, Maturu S, Waldron J, Wang $\mathrm{H}$, et al. Generalized periodic discharges with and without triphasic morphology. J Clin Neurophysiol 2018;35:14450.

24. Kaplan PW. EEG criteria for nonconvulsive status epilepticus. Epilepsia 2007;48 Suppl 8:39-41.

25. Boulanger JM, Deacon C, Lécuyer D, Gosselin S, Reiher J. Triphasic waves versus nonconvulsive status epilepticus: EEG distinction. Can J Neurol Sci 2006;33:175-80.

26. Kaya D, Bingol CA. Significance of atypical triphasic waves for diagnosing nonconvulsive status epilepticus. Epilepsy Behav 2007; 11:567-77.

27. Trinka E, Leitinger M. Which EEG patterns in coma are nonconvulsive status epilepticus? Epilepsy Behav 2015;49:203-22.

28. Rodriguez Ruiz A, Vlachy J, Lee JW, Gilmore EJ, Ayer T, Haider $\mathrm{HA}$, et al. Association of periodic and rhythmic electroencephalographic patterns with seizures in critically ill patients. JAMA Neurol 2017;74:181-8.

29. Husain AM, Mebust KA, Radtke RA. Generalized periodic epileptiform discharges: etiologies, relationship to status epilepticus, and prognosis. J Clin Neurophysiol 1999;16:51-8.

30. Fong MWK, Gaspard N, Hirsch LJ. Generalized periodic discharges with and without triphasic morphology. J Clin Neurophysiol 2019;36:173-4.

31. Bauer G, Trinka E, Kaplan PW. EEG patterns in hypoxic encephalopathies (post-cardiac arrest syndrome): fluctuations, transitions, and reactions. J Clin Neurophysiol 2013;30:477-89.

32. Ruijter BJ, van Putten MJ, Hofmeijer J. Generalized epileptiform discharges in postanoxic encephalopathy: quantitative characterization in relation to outcome. Epilepsia 2015;56:1845-54.

33. Gilmore EJ, Gaspard N, Choi HA, Cohen E, Burkart KM, Chong $\mathrm{DH}$, et al. Acute brain failure in severe sepsis: a prospective study in the medical intensive care unit utilizing continuous EEG monitoring. Intensive Care Med 2015;41:686-94. 
34. Rossetti AO, Oddo M, Liaudet L, Kaplan PW. Predictors of awakening from postanoxic status epilepticus after therapeutic hypothermia. Neurology 2009;72:744-9.

35. Ruijter BJ, van Putten MJ, Horn J, Blans MJ, Beishuizen A, van Rootselaar AF, et al. Treatment of electroencephalographic status epilepticus after cardiopulmonary resuscitation (TELSTAR): study protocol for a randomized controlled trial. Trials 2014;15:433.
36. O'Rourke D, Chen PM, Gaspard N, Foreman B, McClain L, Karakis I, et al. Response rates to anticonvulsant trials in patients with triphasic-wave EEG patterns of uncertain significance. Neurocrit Care 2016;24:233-9.

37. Bermeo-Ovalle A. Triphasic waves: swinging the pendulum back in this diagnostic dilemma. Epilepsy Curr 2017;17:40-2. 